\title{
Special issue on the use of accurate tissue and body models in RF and MW medical applications
}

\author{
Giuseppe Ruvio \\ Technological University Dublin, Giuseppe.Ruvio@tudublin.ie \\ Marta Cavagnaro \\ Sapienza University of Rome
}

Follow this and additional works at: https://arrow.tudublin.ie/ahfrcart

Part of the Systems and Communications Commons

\section{Recommended Citation \\ Ruvio, G. and Cavagnaro, M. (2018), Special issue on the use of accurate tissue and body models in RF and MW medical applications. Int J RF Microw Comput Aided Eng, 28:. DOI: 10.1002/mmce.21264}

This Article is brought to you for free and open access by the Antenna \& High Frequency Research Centre at ARROW@TU Dublin. It has been accepted for inclusion in Articles by an authorized administrator of ARROW@TU Dublin. For more information, please contact arrow.admin@tudublin.ie, aisling.coyne@tudublin.ie, gerard.connolly@tudublin.ie.

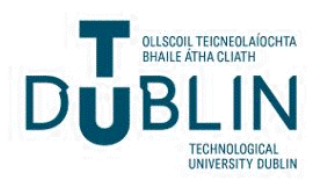




\section{Special issue on the use of accurate tissue and body models in RF and MW medical applications}

Medical applications of electromagnetic fields are emerging as new options for diagnosis and therapy of several diseases. Examples are microwave (MW) resonance imaging, ultrawideband (UWB) radars for the detection and monitoring of the respiratory or cardiac activities, thermal therapies for the minimally invasive treatment of tumors. Among the emerging technologies, miniaturized sensors and antennas to be implanted inside the human body have been pivotal for ground-breaking medical telemetry and telemedicine. Additionally, promising results have been obtained by MW imaging for the diagnosis of cancer disease, which looks for tumors based on the different dielectric properties of the diseased tissue with respect to the healthy one. Within therapeutic scenarios, radio frequency (RF) and MW Ablation, as well as electromagnetic hyperthermia, have been proven successful in a broad range of clinical treatments.

Great enthusiasm and expectations were posed on these emerging applications, but their positive aspects and advancements with respect to the already well-established techniques need to be proved. In this respect, accurate models of the human anatomy including the target tissue and detailed knowledge of the dielectric properties of human tissues are needed and should be developed and validated. These models would be the base for validation studies, as well as for the development of treatment planning procedures to allow definition of the "electromagnetic dose" to be delivered to a patient for the specific pathology.

Accurate modellng assumes a fundamental role in supporting experimental studies, boosting confidence of the medical community towards emerging technologies and supporting therapeutics with reliable pre-treatment planning.

This special issue focuses on the current state of the art of tissue and body modeling to support emerging biomedical applications where RF or MW energy is used for diagnosis and/or therapy.

The issue consists of ten papers, which deal with different aspects of body modeling. Although the fundamental need of accurate dielectric properties of biological tissues for reliable modeling and efficient designing is explored in the contributions "Minimum Information for Dielectric Measurements of Biological Tissues (MINDER): A Framework for Repeatable and Reusable Data", "A study of the effects of preservative solutions on the dielectric properties of biological tissue" and "Comparison of different methods for dielectric properties measurements in liquid sample media", the articles "Investigation and Reduction Methods of Specific Absorption Rate (SAR) for Biomedical Applications: a survey" and "Effects of Tissues and Geometric Shapes of Phantoms on the Specific Energy Absorption Rate" focus on the role of dosimetric studies in biomedical electromagnetic engineering. Dosimetric analysis plays a fundamental role in the assessment of hyperthermia treatments as shown in the article "Human body models for validation studies of deep hyperthermia" where the role of accurate body models is considered and in "Towards Enhanced Energy Localization in Wideband Hyperthermia System Using Transmission Line Model" to validate a novel approach, which is alternative to conventional single-frequency technology.

Multi-physics modeling is a powerful tool that can enable efficient numerical validation of $\mathrm{MW}$ thermal ablation devices before expensive prototype iterations as shown in "Analysis of Microwave Ablation Antenna Optimization Techniques" or difficult pre-clinical investigation on complex scenarios like in "Numerical evaluation of microwave thermal ablation to treat small adrenocortical masses".

Finally, a novel detection technique in the framework of MW-based biomedical diagnostics is introduced in the paper "S-Band Five-Port Ring Reflectometer-Probe System for In Vitro Breast Tumour Detection".

The broad range of RF and MW biomedical applications explored in this special issue testifies the vibrant research activity and commercial interest on innovative devices for the medtech sector and the vital support of advanced numerical modeling to address the consequent engineering challenges.

\footnotetext{
Giuseppe Ruvio ${ }^{1,2}$, Marta Cavagnaro ${ }^{3}$

${ }^{1}$ College of Engineering and Informatics, National University of Ireland Galway, Ireland

${ }^{2}$ Antenna \& High Frequency Research Centre, Dublin Institute of

Technology, Ireland

${ }^{3}$ Dept. of Information Engineering, Electronics and Telecommunications, Sapienza University, Rome, Italy
} 